\title{
Susceptibility of Cucurbita spp to the cucurbit root-rot fungus, Fusarium solani f sp cucurbitae race 1
}

\author{
H Nagao ${ }^{1 *}, \mathrm{~K} \mathrm{Sato}^{2}, \mathrm{~S}$ Ogiwara $^{2}$ \\ ${ }^{1}$ Faculty of Horticulture, Chiba University, Matsudo 271; \\ ${ }^{2}$ Nihon Horticultural Production Institute (Enken), Matsudo 271, Japan
}

(Received 15 May 1993; accepted 13 December 1993)

\begin{abstract}
Summary - We examined the reactions of Cucurbita spp to Fusarium root rot by evaluating disease severity. Cucurbit plants exhibited different susceptibilities to isolates of Fusarium solani f $\mathrm{sp}$ cucurbitae race 1. Cultivars 'Suzunari-nishiki' and 'Miyako' of Cucurbita maxima were more susceptible than $C$ pepo 'Zam Squash' and $C$ moschata 'Yashiro-yon-go'. The aggressiveness of the isolates to cucurbits also varied. Perithecia were formed in some dual cultures between field isolates. All perithecia were fertile. The possibilities for the emergence of new races or virulent strains are discussed.
\end{abstract}

Fusarium solani f sp cucurbitae/cucurbit root rot/cucurbit cultivars/disease severity/perithecia

Résumé - Sensibilité de Cucurbita spp à la pourriture racinaire due au Fusarium solani $\mathrm{f}$ sp cucurbitae. La fusariose de pourriture des racines de Cucurbitacées affecte gravement les cultures de Cucurbita spp au Japon. Aucun moyen de lutte n'étant parfaitement satisfaisant, il est intéressant de déterminer la sensibilité variétale de quelques cultivars à cette maladie. Six souches de $\mathrm{F}$ solani isolées de plantes malades ont été caractérisées et déterminées comme appartenant à la forme spéciale cucurbitae. Leur pouvoir pathogène a été évalué sur 5 cultivars de Cucurbita spp après infestation artificielle du sol. Les résultats indiquent que toutes les souches ne présentent pas exactement la même virulence sur les plantes et réagissent différemment à l'inoculation, 'Yashiro-yon-go' de Cucurbita moschata étant plus tolérant que les cultivars de Cucurbita maxima. La capacité de certains isolats de $\mathrm{F}$ solani à former des périthèces fertiles permet d'expliquer cette variabilité du pouvoir pathogène des souches et laisse craindre l'apparition de races nouvelles.

Fusarium solani $f$ sp cucurbitae / pourriture des racines/cultivars de Cucurbita / reproduction sexée

\section{INTRODUCTION}

Soil-borne diseases are difficult to control even when effective chemicals are available. In soil-borne diseases, vascular wilts can be controlled by using resistant cultivars and grafting susceptible varieties onto resistant root stocks. In the case of Fusarium wilt of cucumber, cucumber plants have been grafted onto Cucurbita ficifolia 'Kurodane'. However, grafting does not control the root rot of cucurbit, which was first observed in Okinawa, Japan in 1978, and Fusarium solani $f \mathrm{sp}$ cucurbitae race 1 was identified (Kinjo et al, 1987). This was the first case recorded in Japan since this pathogen was reported to infect cucurbit in South Africa (Doidge, 1938) and was described as Fusarium solani in USA in 1941 (Snyder and Hansen, 1941).

\footnotetext{
* Correspondence and reprints
} 
Okinawa isolates found on cucumber ( $\mathrm{Cu}$ cumis sativus $\mathrm{L}$ ) grafted onto balsam pear (Momordica charantia L) attacked cucumber, bonnet gourd (Luffa cylindrica Roem) and watermelon (Citrullus vulgaris Schrad) in greenhouse tests (Kinjo et al, 1989). In 1987, this root rot occurred in the geographically isolated regions of Okayama (Ohto et al, 1989; Kasuyama et al, 1990) and Ibaraki (Shimonagane, 1989; Shimonagane et al, 1989).

All varieties of cucurbits were susceptible to Fusarium root rot in greenhouse tests (Toussoun and Snyder, 1961). Recently, various species, including cultivars of cucurbits, were re-examined for susceptibility (Vannacci and Gambogi, 1980; Paternotte, 1987). Although highly resistant species and cultivars have not been detected, it is necessary to find resistant breeding materials.

In this paper, we report on the virulence of 6 different isolates of $F$ solani $f \mathrm{sp}$ cucurbitae and the degree of susceptibility of cucurbit seedlings to root rot.

\section{MATERIALS AND METHODS}

\section{Morphology of isolates}

Fruit rot of winter squash (Cucurbita maxima Duch 'Ebisu') was found in the field of Nihon Horticultural Production Institute (Enken) in 1988. Both large and small lesions were observed. Six isolates were obtained. In addition, another (NW-1) was isolated from an imported winter squash fruit 'Ebisu' from New Zealand. Spore length and width for all isolates were compared with Fusarium solani $f \mathrm{sp}$ cucurbitae race 1 .

\section{Pathogenicity to cucurbit fruit}

To determine the individual form of the isolates, their pathogenicity was examined on fruits of 'Ebisu'. The isolates were cultured on potato sucrose agar (PSA; $200 \mathrm{~g}$ potato, $20 \mathrm{~g}$ sucrose, $17 \mathrm{~g}$ agar, and distilled water to $1 \mathrm{l}$ ) for $14 \mathrm{~d}$ in the dark at $28^{\circ} \mathrm{C}$ and disks were cut with a $10-\mathrm{mm}$ diameter cork bore. These disks were fixed with adhesive tape on the upper and middle parts of the fruits without wounding them. Inoculated fruits were incubated at $25^{\circ} \mathrm{C}$ in plastic bags to avoid contamination. The progress of symptoms was observed daily.

\section{Pathogenecity to seedlings of cucurbit varieties}

The pathogenicity of 6 isolates was tested on 5 different cultivars of cucurbits and 1 of cucumber 'Ootone-ichi-go'. The cultivars examined were: $\mathrm{Cu}$ curbita maxima 'Suzunari-nishiki', 'Miyako' and 'Akazukin'; $C$ pepo 'Zam Squash'; and C moschata 'Yashiro-yon-go'. Spore suspensions were prepared from cultures grown on PSA for $14 \mathrm{~d}$ at $28^{\circ} \mathrm{C}$ and added at a concentration of $1 \times 10^{4} \mathrm{cfu} / \mathrm{g}$ of dry soil. Steamed soil was potted in $18-\mathrm{cm}$ diameter glazed pots. The seeds were surface disinfected in 5\% sodium hypochlorite solution for $5 \mathrm{~min}$, rinsed with running tap water and sown when they germinated. The plants were grown in the greenhouse. The average air and soil temperatures during the experiment were $27.0^{\circ} \mathrm{C}$ and $24.7^{\circ} \mathrm{C}$, respectively. The severity of seedling invasion was determined after $40 \mathrm{~d}$ according to the rating scale in fig 1 . Disease severity was defined as follows: 0: healthy, no infection; 1: hypocotyl is healthy but slight discoloration is found on the root; 2: hypocotyl is healthy but $10 \%$ of the root is discolored; 3 : hypocotyl is slightly infected and $30 \%$ of the root and secondary root are discolored; 4: hypocotyl is infected and $70 \%$ of the root and secondary root are discolored; and 5: hypocotyl is heavily infected and the whole root is discolored, or the plant is dead. The disease index (DI) was calculated from the formula noted below.

$$
\begin{aligned}
\mathrm{DI}=\left(0 \times n_{0}\right)+\left(1 \times n_{1}\right)+\left(2 \times n_{2}\right) & +\left(3 \times n_{3}\right) \\
& +\left(4 \times n_{4}\right)+\left(5 \times n_{5}\right) / N
\end{aligned}
$$

where $N$ indicates number of plants examined and $n_{0}$ to $n_{5}$ are the number of plants corresponding to each DS of 0 to 5 .

All roots and hypocotyls with distinct rot symptoms (DI > 3) were placed on water agar (WA) in an attempt to isolate the pathogen. When seedlings damped off, their roots and hypocotyls were observed and cultured on WA to determine the causal agents.

\section{Perithecia formation between isolates}

Perithecia formation was examined after pairing each isolate with others and with 3 individual forms (phaseoli, pisi and radicola) of Fusarium solani. The fungi were cultured on PSA for $5 \mathrm{~d}$ in

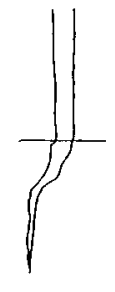

o

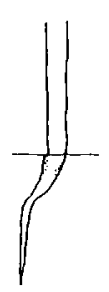

I

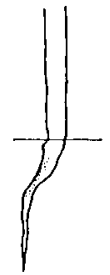

2

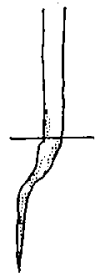

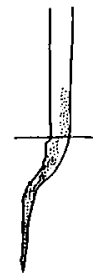

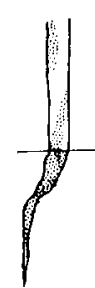

5
Fig 1. Examples of disease severity (DS) for root-rot of cucurbit caused by Fusarium solani $\mathrm{F}$ sp cucurbitae race 1 . 
the dark at $28^{\circ} \mathrm{C}$ before disks were cut from the margin of colonies with a $5-\mathrm{mm}$ diameter cork bore. These disks were paired with each other on PSA at $28^{\circ} \mathrm{C}$ under continuous light. After 4 months of incubation, these cultures were transferred to the laboratory bench. After another 2 months, perithecia were observed in certain pairings.

\section{RESULTS}

\section{Characterization of the Japanese isolates of $\mathrm{F}$ solani from cucurbits}

Macroconidia did not form on either PSA or Carnation leaf agar (CLA). However, macroconidia were observed when the infected root segments were incubated on water agar $(2 \%)$ at room temperature. Five-septate macroconidia were dominant and the conidial size ranged from $37.5-55.0 \mu \mathrm{m} \times 3.8-5.0 \mu \mathrm{m}$ (fig 2a, 2b) Microconidia were observed on both PSA and CLA. They were formed in false heads on long, branched phialides (fig 2c). Chlamydospores were formed intercalary on the hyphae. The colonies were ivory to beige and grew radially.

Symptons of water-soaking were observed around the disks of isolate P1-3 after $9 \mathrm{~d}$ incubation but soft rot of fruits only appeared after $17 \mathrm{~d}$ for all isolates (fig 3 ). In addition, these isolates also invaded cucurbit seedlings as described below. Based on the morphological characters and pathogenicity, these isolates were identified as Fusarium solani $f \mathrm{sp}$ cucurbitae race 1 (Toussoun and Snyder, 1961).

\section{Pathogenicity to cucurbit seedlings}

The degree of susceptibility of the cucurbits varied (table 1). 'Suzunari-nishiki' was severely affected by all isolates with a DI of approximately 4.0. The cultivars 'Suzunari-nishiki' and 'Miyako' were the most severely affected by isolates A-3 and NW-1. Over $20 \%$ of root segments were discolored entirely $(D I=5)$ on 'Suzunari-nishiki'. 'Miyako' was also severely affected by isolates A-1, A-3, B-1 and NW-1, but was not severely affected by the isolates $\mathrm{P} 1-2$ and $\mathrm{P} 1-3(\mathrm{DI}=3.30$ and
2.65 , respectively). 'Akazukin' was less susceptible to the isolates $A-1, A-3, B-1$ and NW1. $C$ pepo 'Zam Squash' and $C$ moschata 'Yashiro-yon-go' were less susceptible than $C$ maxima. These cultivars were severely affected only by isolates B-1, P1-3 and NW-1 (fig 5). The disease indices for these cultivars showed a discontinuity for infection. Cucumber 'Ootone-ichi-go' was also less susceptible to these isolates except for isolate P1-2.

The frequency of reisolation from the cultivars of $C$ maxima was greater than $80 \%$ of infected plants (table I). When severely infected hypocotyls or roots were examined, secondary invaders sometimes impeded the growth of $F$ solani. On the contrary, all of the pieces of $C$ pepo and $C$ moschata yielded colonies of $F$ solani. Although these pieces were slightly infected, these symptoms were in no doubt caused by $F$ solani.

Infectivities of our isolates were compared between cucurbit plants (table II). Virulences of the 6 isolates were significantly different. Isolates $\mathrm{A}-1, \mathrm{~A}-3, \mathrm{~B}-1$ and $\mathrm{NW}-1$ severely affected 3 cultivars of $C$ maxima. Isolates $\mathrm{P} 1-2$ and P1-3 moderately affected all cucurbits examined. Average disease indices indicated that the values of DI were related to host varieties. Host plants were divided into 3 groups based on disease severity: $C$ maxima 'Suzunari-nishiki' and 'Miyako' were the most susceptible; $C$ maxima 'Akazukin' and $C$ pepo 'Zam Squash' were intermediate; and $C$ moschata 'Yashiro-yon-go' and Cucumis sativus 'Oostone-ichi-go' were the least susceptible. This grouping was supported by statistical analysis (table II).

\section{Perithecia formation between isolates}

Perithecia formed in the pairings of $A-1 / P 1-2$, A-2/P1-3, A-3/P1-2, A-3/P1-3, A-3/NW-1 and P1-3/B-1 (fig 4; table III). Perithecia formed on both colonies. A few perithecia formed at the line of contact between the colonies. No perithecia or perithecial initials were observed in pairings with the different individual forms of $F$ solani. In pairings with isolate $\mathrm{P} 1$ 3 , perithecia formed on its own colony or on the opposite colony but never on both. This result indicated that the isolate $\mathrm{P} 1-3$ is a hermaphrodite but self-incompatible strain. 


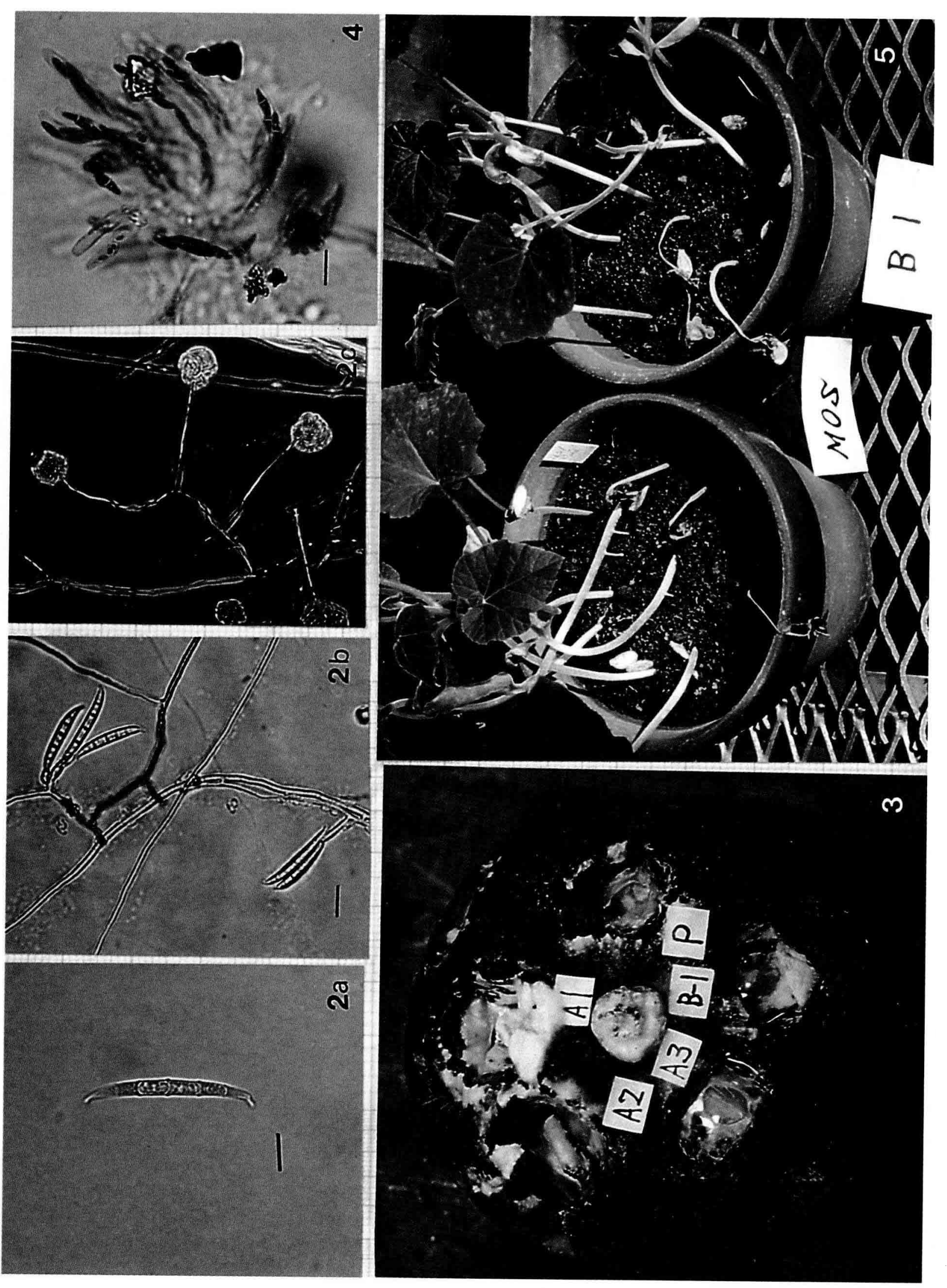

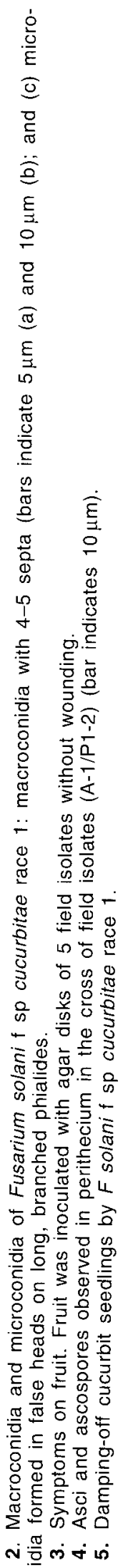

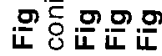


Table I. Comparison of disease severity and reisolation frequency between cucurbit plants for 6 isolates of Fusarium solani f $\mathrm{sp}$ cucurbitae.

\begin{tabular}{|c|c|c|c|c|c|c|c|c|c|c|c|c|}
\hline \multirow[t]{2}{*}{ Host plant } & \multirow[t]{2}{*}{ Isolate } & \multicolumn{9}{|c|}{$\begin{array}{l}\text { Disease severity and percentage of diseased } \\
\text { plants }\end{array}$} & \multicolumn{2}{|c|}{ Reisolation } \\
\hline & & $\begin{array}{l}\text { No of } \\
\text { plants }\end{array}$ & 0 & Dis & eas & 3 & idex & 5 & Average & $\begin{array}{l}\text { Diseased } \\
\text { plants }(\%)\end{array}$ & $\begin{array}{l}\text { No of } \\
\text { plants }\end{array}$ & $\begin{array}{l}\text { Average } \\
(\%)\end{array}$ \\
\hline $\begin{array}{l}\text { Cucurbita maxima cv } \\
\text { Suzunari-nishiki }\end{array}$ & $\begin{array}{l}A-1 \\
A-3 \\
B-1 \\
P 1-2 \\
P 1-3 \\
N W-1\end{array}$ & $\begin{array}{l}19 \\
19 \\
19 \\
20 \\
15 \\
18\end{array}$ & $\begin{array}{l}0 \\
0 \\
0 \\
0 \\
0 \\
0\end{array}$ & $\begin{array}{l}0 \\
0 \\
0 \\
0 \\
0 \\
0\end{array}$ & $\begin{array}{l}2 \\
0 \\
0 \\
0 \\
1 \\
0\end{array}$ & $\begin{array}{l}7 \\
1 \\
7 \\
6 \\
6 \\
0\end{array}$ & $\begin{array}{l}3 \\
7 \\
8 \\
9 \\
2 \\
7\end{array}$ & $\begin{array}{r}7 \\
11 \\
4 \\
5 \\
6 \\
11\end{array}$ & $\begin{array}{l}3.79^{\mathrm{a}} \\
4.53^{\mathrm{ab}} \\
3.84^{\mathrm{ab}} \\
3.95^{\mathrm{ab}} \\
3.87^{\mathrm{ab}} \\
4.61^{\mathrm{b}}\end{array}$ & $\begin{array}{l}100.0^{*} \\
100.0 \\
100.0 \\
100.0 \\
100.0 \\
100.0\end{array}$ & $\begin{array}{l}16 \\
18 \\
20 \\
17 \\
16 \\
16\end{array}$ & $\begin{array}{r}93.8 \\
94.4 \\
95.0 \\
100.0 \\
93.8 \\
100.0\end{array}$ \\
\hline $\begin{array}{l}\text { Cucurbita maxima cv } \\
\text { Miyako }\end{array}$ & $\begin{array}{l}\text { A-1 } \\
\text { A-3 } \\
\text { B-1 } \\
\text { P1-2 } \\
\text { P1-3 } \\
\text { NW-1 }\end{array}$ & $\begin{array}{l}20 \\
19 \\
20 \\
20 \\
20 \\
20\end{array}$ & $\begin{array}{l}0 \\
0 \\
0 \\
0 \\
0 \\
0\end{array}$ & $\begin{array}{l}0 \\
0 \\
0 \\
0 \\
1 \\
0\end{array}$ & $\begin{array}{l}0 \\
0 \\
2 \\
4 \\
8 \\
0\end{array}$ & $\begin{array}{r}9 \\
2 \\
8 \\
10 \\
9 \\
1\end{array}$ & $\begin{array}{l}4 \\
7 \\
5 \\
2 \\
1 \\
2\end{array}$ & $\begin{array}{r}7 \\
10 \\
5 \\
4 \\
1 \\
17\end{array}$ & $\begin{array}{l}3.90^{\mathrm{bc}} \\
4.42^{\mathrm{cd}} \\
3.65^{\mathrm{b}} \\
3.30^{\mathrm{ab}} \\
2.65^{\mathrm{a}} \\
4.80^{\mathrm{d}}\end{array}$ & $\begin{array}{l}100.0^{\star} \\
100.0 \\
100.0 \\
100.0 \\
100.0 \\
100.0\end{array}$ & $\begin{array}{l}15 \\
17 \\
15 \\
14 \\
13 \\
18\end{array}$ & $\begin{array}{r}86.7 \\
100.0 \\
93.3 \\
100.0 \\
92.3 \\
94.4\end{array}$ \\
\hline $\begin{array}{l}\text { Cucurbita maxima cv } \\
\text { Akazukin }\end{array}$ & $\begin{array}{l}\text { A-1 } \\
\text { A-3 } \\
\text { B-1 } \\
\text { P1-2 } \\
\text { P1-3 } \\
\text { NW-1 }\end{array}$ & $\begin{array}{l}20 \\
20 \\
18 \\
19 \\
20 \\
20\end{array}$ & $\begin{array}{l}0 \\
0 \\
0 \\
0 \\
0 \\
2\end{array}$ & $\begin{array}{r}2 \\
2 \\
1 \\
0 \\
10 \\
4\end{array}$ & $\begin{array}{l}6 \\
4 \\
2 \\
3 \\
1 \\
0\end{array}$ & $\begin{array}{r}7 \\
9 \\
8 \\
10 \\
7 \\
5\end{array}$ & $\begin{array}{l}3 \\
1 \\
7 \\
4 \\
1 \\
0\end{array}$ & $\begin{array}{l}2 \\
4 \\
0 \\
2 \\
1 \\
9\end{array}$ & $\begin{array}{l}2.85^{\mathrm{a}} \\
3.05^{\mathrm{a}} \\
3.17^{\mathrm{a}} \\
2.84^{\mathrm{a}} \\
2.10^{\mathrm{a}} \\
3.20^{\mathrm{a}}\end{array}$ & $\begin{array}{c}100.0^{\star} \\
100.0 \\
100.0 \\
100.0 \\
100.0 \\
90.0\end{array}$ & $\begin{array}{r}13 \\
15 \\
16 \\
13 \\
9 \\
14\end{array}$ & $\begin{array}{r}92.3 \\
80.0 \\
93.8 \\
100.0 \\
88.9 \\
92.9\end{array}$ \\
\hline $\begin{array}{l}\text { Cucurbita pepo cv } \\
\text { Zam Squash }\end{array}$ & $\begin{array}{l}\text { A-1 } \\
\text { A-3 } \\
\text { B-1 } \\
\text { P1-2 } \\
\text { P1-3 } \\
\text { NW-1 }\end{array}$ & $\begin{array}{l}19 \\
\text { NT } \\
20 \\
19 \\
19 \\
10\end{array}$ & $\begin{array}{l}0 \\
- \\
0 \\
1 \\
2 \\
0\end{array}$ & $\begin{array}{l}2 \\
- \\
2 \\
3 \\
6 \\
0\end{array}$ & $\begin{array}{c}4 \\
- \\
10 \\
10 \\
8 \\
4\end{array}$ & $\begin{array}{l}12 \\
- \\
7 \\
5 \\
0 \\
6\end{array}$ & $\begin{array}{l}0 \\
- \\
0 \\
0 \\
0 \\
0\end{array}$ & $\begin{array}{l}0 \\
- \\
1 \\
0 \\
2 \\
0\end{array}$ & $\begin{array}{c}2.42^{\mathrm{a}} \\
- \\
2.40^{\mathrm{a}} \\
2.00^{\mathrm{a}} \\
1.68^{\mathrm{a}} \\
2.60^{\mathrm{a}}\end{array}$ & $\begin{array}{c}100.0 \\
- \\
100.0 \\
94.7 \\
89.5 \\
100.0\end{array}$ & $\begin{array}{r}6 \\
- \\
14 \\
5 \\
10 \\
6\end{array}$ & $\begin{array}{c}100.0 \\
- \\
100.0 \\
100.0 \\
100.0 \\
100.0\end{array}$ \\
\hline $\begin{array}{l}\text { Cucurbita moschata cv } \\
\text { Yashiro-yon-go }\end{array}$ & $\begin{array}{l}A-1 \\
A-3 \\
B-1 \\
\text { P1-2 } \\
\text { P1-3 } \\
\text { NW-1 }\end{array}$ & $\begin{array}{l}17 \\
20 \\
17 \\
10 \\
19 \\
16\end{array}$ & $\begin{array}{l}2 \\
2 \\
2 \\
0 \\
0 \\
8\end{array}$ & $\begin{array}{l}7 \\
7 \\
6 \\
1 \\
6 \\
3\end{array}$ & $\begin{array}{r}7 \\
10 \\
4 \\
4 \\
11 \\
2\end{array}$ & $\begin{array}{l}1 \\
1 \\
0 \\
5 \\
1 \\
1\end{array}$ & $\begin{array}{l}0 \\
0 \\
0 \\
0 \\
0 \\
0\end{array}$ & $\begin{array}{l}0 \\
0 \\
5 \\
0 \\
1 \\
2\end{array}$ & $\begin{array}{l}1.41^{\mathrm{a}} \\
1.50^{\mathrm{a}} \\
2.29^{\mathrm{a}} \\
2.40^{\mathrm{a}} \\
1.89^{\mathrm{a}} \\
1.25^{\mathrm{a}}\end{array}$ & $\begin{array}{r}88.2 \\
90.0 \\
88.2 \\
100.0 \\
100.0 \\
50.0\end{array}$ & $\begin{array}{l}1 \\
5^{\star \star} \\
4 \\
5 \\
5^{\star \star} \\
4^{* *}\end{array}$ & $\begin{array}{l}100.0 \\
100.0 \\
100.0 \\
100.0 \\
100.0 \\
100.0\end{array}$ \\
\hline $\begin{array}{l}\text { Cucumis sativus cv } \\
\text { Ootone-ichi-go }\end{array}$ & $\begin{array}{l}A-1 \\
A-3 \\
B-1 \\
\text { P1-2 } \\
\text { P1-3 } \\
\text { NW-1 }\end{array}$ & $\begin{array}{l}19 \\
20 \\
20 \\
20 \\
20 \\
20\end{array}$ & $\begin{array}{r}5 \\
7 \\
1 \\
5 \\
16 \\
8\end{array}$ & $\begin{array}{l}3 \\
3 \\
4 \\
3 \\
0 \\
7\end{array}$ & $\begin{array}{r}4 \\
10 \\
8 \\
5 \\
3 \\
4\end{array}$ & $\begin{array}{l}6 \\
0 \\
6 \\
1 \\
1 \\
1\end{array}$ & $\begin{array}{l}1 \\
0 \\
1 \\
1 \\
0 \\
0\end{array}$ & $\begin{array}{l}0 \\
0 \\
0 \\
5 \\
0 \\
0\end{array}$ & $\begin{array}{l}1.74^{\mathrm{a}} \\
1.15^{\mathrm{a}} \\
2.10^{\mathrm{a}} \\
2.15^{\mathrm{a}} \\
0.45^{\mathrm{a}} \\
0.90^{\mathrm{a}}\end{array}$ & $\begin{array}{l}73.7 \\
65.0 \\
95.0 \\
75.0 \\
20.0 \\
60.0\end{array}$ & $\begin{array}{l}10 \\
\text { NT } \\
\text { NT } \\
\text { NT } \\
\text { NT } \\
\text { NT }\end{array}$ & $\begin{array}{c}50.0 \\
- \\
- \\
- \\
- \\
-\end{array}$ \\
\hline
\end{tabular}

NT = not tested; disease index was determined with the diagrams in fig 1 and calculated with the formula; for each isolate, values within a column followed by different letters are significantly different at $P=0.01$ according to Duncan's multiple range test; " plants with a disease index of 1 or greater; "* as there were few plants with a DI rating of $>3$, the plants with $\mathrm{DI}=2$ were also used for reisolation.

\section{DISCUSSION}

The symptoms of cucurbit root-rot are characterized by rotting of hypocotyl and root and also dry rot on fruit. Four or 5 septated macroconidia are known as a significant key for determination of race. These features have previously been reported in the literature (Vannacci and Gambogi, 1980; Hall et al, 1981). When estimating disease severity, it is easy to determine the number of 'diseased' or 'infected' fruits because fruit rot begins with tissue degradation. Even small water- 
Table II. Comparison of infectivity of 6 isolates of Fusarium solani $f \mathrm{sp}$ cucurbitae for cucurbit plants.

\begin{tabular}{|c|c|c|c|}
\hline Isolate & Host plant & No of plants & $\begin{array}{c}\text { Average } \\
D I\end{array}$ \\
\hline$A-1$ & $\begin{array}{l}\text { Cucurbita maxima cv Suzunari-nishiki } \\
\text { Cucurbita maxima cv Miyako } \\
\text { Cucurbita maxima cv Akazukin } \\
\text { Cucurbita pepo cv Zam Squash } \\
\text { Cucurbita moschata cv Yashiro-yon-go } \\
\text { Cucumis sativus cv Ootone-ichi-go }\end{array}$ & $\begin{array}{l}19 \\
20 \\
20 \\
19 \\
17 \\
19\end{array}$ & $\begin{array}{l}3.79^{\mathrm{c}} \\
3.90^{\mathrm{c}} \\
2.85^{\mathrm{b}} \\
2.42^{\mathrm{ab}} \\
1.41^{\mathrm{a}} \\
1.74^{\mathrm{a}}\end{array}$ \\
\hline$A-3$ & $\begin{array}{l}\text { Cucurbita maxima cv Suzunari-nishiki } \\
\text { Cucurbita maxima cv Miyako } \\
\text { Cucurbita maxima cv Akazukin } \\
\text { Cucurbita pepo cv Zam Squash } \\
\text { Cucurbita moschata cv Yashiro-yon-go } \\
\text { Cucumis sativus cv Ootone-ichi-go }\end{array}$ & $\begin{array}{l}19 \\
19 \\
20 \\
\text { NT } \\
20 \\
20\end{array}$ & $\begin{array}{l}4.53^{\mathrm{c}} \\
4.42^{\mathrm{c}} \\
3.05^{\mathrm{b}} \\
- \\
1.50^{\mathrm{a}} \\
1.15^{\mathrm{a}}\end{array}$ \\
\hline B-1 & $\begin{array}{l}\text { Cucurbita maxima cv Suzunari-nishiki } \\
\text { Cucurbita maxima cv Miyako } \\
\text { Cucurbita maxima cv Akazukin } \\
\text { Cucurbita pepo cv Zam Squash } \\
\text { Cucurbita moschata cv Yashiro-yon-go } \\
\text { Cucumis sativus cv Ootone-ichi-go }\end{array}$ & $\begin{array}{l}19 \\
20 \\
18 \\
20 \\
17 \\
20\end{array}$ & $\begin{array}{l}3.84^{c} \\
3.65^{b c} \\
3.17^{a b c} \\
2.40^{a b c} \\
2.29^{a b} \\
2.10^{a}\end{array}$ \\
\hline$P_{1-2}$ & $\begin{array}{l}\text { Cucurbita maxima cv Suzunari-nishiki } \\
\text { Cucurbita maxima cv Miyako } \\
\text { Cucurbita maxima cv Akazukin } \\
\text { Cucurbita pepo cv Zam Squash } \\
\text { Cucurbita moschata cv Yashiro-yon-go } \\
\text { Cucumis sativus cv Ootone-ichi-go }\end{array}$ & $\begin{array}{l}20 \\
20 \\
19 \\
19 \\
10 \\
20\end{array}$ & $\begin{array}{l}3.95^{\mathrm{a}} \\
3.30^{\mathrm{a}} \\
2.84^{\mathrm{a}} \\
2.00^{\mathrm{a}} \\
2.40^{\mathrm{a}} \\
2.15^{\mathrm{a}}\end{array}$ \\
\hline P1-3 & $\begin{array}{l}\text { Cucurbita maxima cv Suzunari-nishiki } \\
\text { Cucurbita maxima cv Miyako } \\
\text { Cucurbita maxima cv Akazukin } \\
\text { Cucurbita pepo cv Zam Squash } \\
\text { Cucurbita moschata cv Yashiro-yon-go } \\
\text { Cucumis sativus cv Ootone-ichi-go }\end{array}$ & $\begin{array}{l}15 \\
20 \\
20 \\
19 \\
19 \\
20\end{array}$ & $\begin{array}{l}3.87^{\mathrm{c}} \\
2.65^{\mathrm{b}} \\
2.10^{\mathrm{b}} \\
1.68^{\mathrm{b}} \\
1.89^{\mathrm{b}} \\
0.45^{\mathrm{a}}\end{array}$ \\
\hline$N W-1$ & $\begin{array}{l}\text { Cucurbita maxima cv Suzunari-nishiki } \\
\text { Cucurbita maxima cv Miyako } \\
\text { Cucurbita maxima cv Akazukin } \\
\text { Cucurbita pepo cv Zam Squash } \\
\text { Cucurbita moschata cv Yashiro-yon-go } \\
\text { Cucumis sativus cv Ootone-ichi-go }\end{array}$ & $\begin{array}{l}18 \\
20 \\
20 \\
10 \\
16 \\
20\end{array}$ & $\begin{array}{l}4.61^{\mathrm{b}} \\
4.80^{\mathrm{b}} \\
3.20^{\mathrm{b}} \\
2.60^{\mathrm{ab}} \\
1.25^{\mathrm{a}} \\
0.90^{\mathrm{a}}\end{array}$ \\
\hline
\end{tabular}

NT = Not tested; disease index was determined with the diagrams in fig 1 and calculated with the formula; for each isolate, values within a column followed by different letters are significantly different at $P=0.01$ according to Duncan's multiple range test.

Table III. Perithecia formation among the different isolates of Fusarium solani.

\begin{tabular}{|c|c|c|c|c|c|c|c|c|c|c|c|}
\hline \multicolumn{2}{|c|}{ Isolates } & $A-1$ & $A-2$ & $A-3$ & $B-1$ & $P l-2$ & $P /-3$ & $N W-1$ & 12200 & 12300 & 12400 \\
\hline f sp cucurbitae & $A-1$ & - & - & - & - & + & - & - & - & - & - \\
\hline & $A-2$ & & - & - & - & - & + & - & - & - & - \\
\hline & $A-3$ & & & - & - & + & + & + & - & - & - \\
\hline & B-1 & & & & - & - & + & - & - & - & - \\
\hline & $\mathrm{Pl}-2$ & & & & & - & - & - & - & - & - \\
\hline & $\mathrm{PI}-3$ & & & & & & - & - & - & - & - \\
\hline & NW-1 & & & & & & & - & - & - & - \\
\hline f sp phaseoli & 12000 & & & & & & & & - & - & - \\
\hline f sp pisi & 12300 & & & & & & & & & - & - \\
\hline $\mathrm{f} \mathrm{sp}$ radicola & 12400 & & & & & & & & & & - \\
\hline
\end{tabular}


soaked symptoms on the fruit are easily recognized as disease. Up to now cucurbit rootrot symptoms have been evaluated as 'infected' or not. However, the significance of infection on the fruits and the seedlings is different. The existence of fruit rot itself means economic loss which may not apply for seedlings. Symptoms on hypocotyls and roots are not necessarily related to the economic importance. For example, some seedlings had severely diseased hypocotyls and roots, while others were very slightly diseased. However, the pathogen could be reisolated from all seedlings. Therefore, seedling root rot is quantitative disease. So the resistance of plants can be compared based on these ratings. We felt this symptom should be assessed with a different method of evaluation than the qualitative one used for fruit rot. Therefore we divided the range of symptoms on hypocotyls and roots into 6 grades of symptoms as shown in fig 1 . This kind of disease index has been used with root rot of beans, caused by $F$ solani $f \mathrm{sp}$ phaseoli $(\mathrm{Fu}$ ruya et al, 1979).

Two cultivars of $C$ maxima, 'Suzunari-nishiki' and 'Miyako', were very susceptible, while 'Akazukin' showed a broad range of symptom development, from severe to slight. The differences between isolates were minimal for cultivars of $C$ maxima which are very susceptible to Fusarium root rot. $C$ pepo and $C$ moschata have been reported to be susceptible to Fusarium root rot (Vannacci and Gambogi, 1980; Paternotte, 1987). However, in our experiments, disease severity on $C$ pepo and $C$ moschata was clearly lower than on $C$ maxima. Furthermore, the number of dead plants was quite small in $C$ pepo and $C$ moschata (table I). In 'Zam Squash' and 'Yashiro-yon-go', some plants damped off within 2 weeks of planting. However, symptoms on surviving plants did not progress to severe lesions when the plants were evaluated $40 \mathrm{~d}$ after planting. Another cultivar, 'Kumi kumi' of $C$ pepo was severely infected by isolate A-3 (Nagao, Sato, Ogiwara, unpublished results). This indicates that the susceptibility of these cultivars may be heterogeneous. These results are interpreted as follows: the inoculum was not distributed evenly or these cultivars are less susceptible to cucurbit root rot. However, at the inoculum density used $\left(10^{4} \mathrm{cfu} / \mathrm{g}\right.$ of dry soil), cultivars of $C$ maxima displayed a high level of infection by the pathogen.
Comparison of infectivities of 6 isolates suggested that isolates A-1, A-3, B-1 and NW-1 were more virulent than isolates $P 1-2$ and $\mathrm{P} 1-3$ (table II). From these results, it was also clearly evident that the virulence of each strain was different. A variability of virulence in $F$ solani $f \mathrm{sp}$ cucurbitae has been reported previously (Prasad, 1949). F solani f sp cucurbitae has a teleomorph (Nectria haematococca). It has been reported that the progeny of 1 cross showed a large degree of variation (Prasad, 1949). Thus it is possible that new virulent strains could appear which may be able to attack resistant cultivars. However, the teleomorph has not been found in nature (Matsuo and Snyder, 1973; Dai et al, 1990). Furthermore, race 1 and race 2 do not cross (Toussoun and Snyder, 1961). F solani species include several strains which can be distinguished from each other by crossing. $N$ haematococca has 3 compatibility types (Matsuo and Snyder, 1973). At lest 1 of our isolates, P1-3, is a self-incompatible hermaphrodite. Our isolates were sampled from the same field at the same time. Only 5 pairings of isolates from the same field were fertile. Although root rot of cucurbit was found in 1978 , intersterile isolates seemed to be differentiated from these isolates. Other strains, which may be differentiated based on their crossing ability, virulence and other physiological characteristics, may appear in Japanese fields.

Incidence of Fusarium fruit rot of cucurbits caused by $F$ solani f sp cucurbitae race 2 has recently increased in the USA. It was reported that weather conditions, especially foggy weather and high humidity, contributed to the high disease incidence (Hall et al, 1981). On the contrary Fusarium root rot has not been noticed during the last $20 \mathrm{yr}$. This situation was supported by the fact that $F$ solani $\mathrm{f} \mathrm{sp}$ cucurbitae race 1 was tested as a biological control agent of the weed, Texas gourd, Cucurbita texana A Gray, which is very susceptible (Weidemann and Templeton, 1988).

Cucurbit fruits play a role in enriching our table. Fortunately race 2 has not appeared in Japan and so we would like to prevent further extension of Fusarium root rot in Japan. It will be necessary to examine the relationships between the inoculum density, disease severity, the environmental conditions and so on. 


\section{ACKNOWLEDGMENTS}

We would like to thank AP Keinath, Clemson University, USA for helpful criticism and advice, C Alabouvette, INRA de Dijon, for revising and translating the abstract, and D Wakatabe for field assistance.

\section{REFERENCES}

Dai K, Kawai A, Nishio T, Goto M (1990) Perithecia formation for Japanese isolates of Fusarium solani sp cucurbitae race 1. Ann Phytopath Soc Jpn 56, 96-97

Doidge EM (1938) Some South African fusaria. Bothalia 3, 331-483

Furuya H, Owada M, Ui T (1979) A suppressive soil of common bean root rot in Kitami district, Hokkaido. Ann Phytopath Soc Jpn 45, 608-617 (in Japanese)

Hall DH, Gubler WD, Sciaroni RH (1981) Fusarium fruit rot of cucurbits. Calif Plant Pathol 54, 3

Kasuyama S, Inoue K, Okamoto $Y$ (1990) Fusarium root rot of Cucurbits occurred in Okayama prefecture. Ann Phytopath Soc Jpn 56, 384

Kinjo K, Tokashiki I, Matsuo T (1987) Fusarium solani $\mathrm{f}$ sp cucurbitae race 1 invading root stocks of withered balsam pear grafted on squash. Ann Phytopath Soc Jpn 53, 86

Kinjo K, Matsuo T, Tokashiki I (1989) Fusarium solani f sp cucurbitae race 1 isolated from root stocks of withered balsam pear grafted on squash. Bull Okinawa Agric Exp Sta 13, 95-98

Matsuo T, Snyder WC (1973) Use of morphology and mating populations in the identification of formae specialis in Fusarium solani. Phytopathology 63, 562-565

Ohto K, Dai K, Shimonagane K (1989) Occurrence and control of cucurbit foot rot caused by $\mathrm{Fu}$ sarium solani $\mathrm{sp}$ cucurbitae race 1. Shokubutuboeki 43, 625-628 (in Japanese)

Paternotte SJ (1987) Pathogenicity of Fusarium solani f sp cucurbitae race 1 to courgette. Neth $J$ Plant Pathol 93, 245-252

Prasad N (1949) Variability of the cucurbit root-rot fungus, Fusarium (Hypomyces) solani f cucurbitae. Phytopathology 39, 133-141

Simonagane K (1989) Occurrence of Fusarium root rot of Cucurbits. Kongetunonouyaku 5, 3033 (in Japanese)

Shimonagane K, Kawamata J, Terunuma S, Matsuda A (1989) Fusarium root rot of Cucurbits occurred in lbaraki prefecture. Ann Phytopath Soc Jpn 55, 120-121

Snyder WC, Hansen HN (1941) The species concept in Fusarium with reference to section Martiella. Amer J Bot 28, 738-742

Toussoun TA, Snyder WC (1961) The pathogenicity, distribution, and control of two races of Fusarium (Hypomyces) solani $f$ cucurbitae. Phytopathology 51, 17-22

Vannacci G, Gambogi P (1980) Fusarium solani $f$ sp cucurbitae razza 1 su semi di Cucurbita pepo $\mathrm{L}$ : reperimento del pathogeno e influenza di condizioni colturali sull'andamento della malattia. Phytopathol Mediterr 19, 103-114

Weidemann GJ, Templeton GE (1988) Efficacy and soil persistence of Fusarium solanif $\mathrm{sp} \mathrm{cu-}$ curbitae for control of Texas gourd (Cucurbita texana). Plant Dis $72,36-38$ 